\title{
Carbon monoxide-releasing molecule-3: Amelioration of renal ischemia reperfusion injury in a rat model
}

\author{
Dae Keun Kim ${ }^{1}$ (i), Su-Jin Shin ${ }^{2}$ (i), Jiyoung Lee ${ }^{3}$ (i), Sung Yul Park ${ }^{3}$ (i), Yong Tae Kim ${ }^{3}$ (i), Hong Yong Choi $^{3}$, \\ Young Eun Yoon ${ }^{3}$ (i), Hong Sang Moon ${ }^{3}$ (i) \\ 'Department of Urology, CHA Fertility Center Seoul Station, CHA University School of Medicine, Seoul, ${ }^{2}$ Department of Pathology, Gangnam Severance Hospital, Yonsei \\ University College of Medicine, Seoul, ${ }^{3}$ Department of Urology, Hanyang University College of Medicine, Seoul, Korea
}

Purpose: Despite the role of carbon monoxide in ameliorating ischemia-reperfusion injury (IRI), its use in the clinical setting is restricted owing to its toxicity. Herein, we investigated the in vivo effects of carbon monoxide-releasing molecule-3 (CORM-3) on IRI. Materials and Methods: Fifteen rats were equally and randomly divided into three groups: sham (right nephrectomy), control (right nephrectomy and left renal ischemia), and CORM-3 (right nephrectomy and CORM-3 injection before left renal ischemia). Kidney tissues and blood samples collected from sacrificed rats were evaluated to determine the renoprotective effect and mechanism of CORM-3.

Results: Concentrations of serum creatinine and kidney injury molecule- 1 in the CORM-3 group were significantly lower than in the control group after 75 minutes of IRI (1.2 vs. $2.4 \mathrm{mg} / \mathrm{dL}, \mathrm{p}=0.01$, and $292 \mathrm{vs.} 550 \mathrm{pg} / \mathrm{mL}, \mathrm{p}<0.001$, respectively). Furthermore, the CORM-3 group exhibited a higher portion of normal tubules and glomeruli. TUNEL staining revealed fewer apoptotic renal tubular cells in the CORM-3 group than in the control group. The expression of 960 genes in the CORM-3 group was also altered. Pretreatment with CORM-3 before renal IRI produced a significant renoprotective effect. Fifteen of the altered genes were found to be involved in the peroxisome proliferator-activated receptors signaling pathway, and the difference in the expression of these genes between the CORM-3 and control groups was statistically significant $(p<0.001)$.

Conclusions: CORM-3 ameliorates IRI by decreasing apoptosis and may be a novel strategy for protection against renal warm IRI.

Keywords: Carbon monoxide; Ischemia; Kidney diseases

This is an Open Access article distributed under the terms of the Creative Commons Attribution Non-Commercial License (http://creativecommons.org/licenses/by-nc/4.0) which permits unrestricted non-commercial use, distribution, and reproduction in any medium, provided the original work is properly cited.

\section{INTRODUCTION}

Ischemia-reperfusion injury (IRI) reduces intracellular levels of ATP and causes ion channel damage leading to cell death [1]. The cells of the renal cortex are vulnerable to changes in oxygen concentration and IRI. The temporary clamping of the renal artery and vein before renal mass excision and renorrhaphy during partial nephrectomy to suture the excised renal tissue can also cause IRI. To prevent IRI during open partial nephrectomy, the kidney is sur-

Received: 23 October, 2019 • Accepted: 5 February, 2020

Corresponding Author: Hong Sang Moon (iD https://orcid.org/0000-0003-2101-1019

Department of Urology, Hanyang University Guri Hospital, Hanyang University College of Medicine, 153 Gyeongchun-ro, Guri 11923, Korea

TEL: +82-31-560-2374, FAX: +82-31-560-2377, E-mail: moonuro@hanyang.ac.kr 
rounded by ice-cold saline packs at the start of the procedure (cold ischemia) [2]. However, recent advances in minimally invasive surgical techniques have made it difficult and time consuming to place ice inside the abdominal cavity during laparoscopic or robotic partial nephrectomy. Warm IRI can be prevented or minimized if the ischemia time is less than 25 minutes [3]. However, a recent study showed that, despite limiting the ischemia time to less than 20 minutes, the risk of renal injury increases by $6 \%$ for every 1-minute increase in ischemia time [4]. Even a single instance of acute renal injury can increase the risk for chronic renal failure by $40 \%$. The frequency of partial nephrectomy has increased geometrically in recent years and currently accounts for over $70 \%$ of all renal tumor surgeries. In addition, as life expectancy has increased, the importance of preserving renal function after the surgical removal of renal tumors has increased. Indeed, a recent analysis of surgical outcomes for kidney cancer evaluated the importance of functional outcomes [5].

In addition to partial nephrectomy, IRI can occur during renal transplantation. The donor kidney is in a state of ischemia until anastomosis with the recipient blood vessels, and injury can occur during reperfusion after anastomosis. Moreover, methods more effective than low-temperature ice storage of the donor kidney during transit have not yet been developed [6]. The role of carbon monoxide (CO) in renal IRI has been a subject of interest since the 2000s [7]. However, there has been limited animal study of the role of $\mathrm{CO}$ in IRI in the kidney. One study demonstrated that inhalation of low-dose $\mathrm{CO}$ together with anaesthetic gas can improve renal function in porcine kidney transplantation recipients. The authors suggested inflammatory modulation as a mechanism for the effects of $\mathrm{CO}$ [8]. Another study demonstrated that the oxygen-binding capacity of $\mathrm{CO}$ is approximately 40fold that of hemoglobin, identifying $\mathrm{CO}$ as a molecule that can deliver oxygen to ischemic organs [7]. Therefore, attention has turned to CO-releasing molecule (CORM). A CORM compound was first developed to deliver $\mathrm{CO}$ to biological target organs by researchers in the United Kingdom (UK) [9,10]. There are several subtypes of CORM; however, CORM3 shows excellent water solubility and rapidly releases CO in physiologic fluids [10]. Therefore, in this study, we aimed to investigate the effects of CORM-3 on IRI of the kidney, prove its mechanism of action, and explore its potential for clinical application.

\section{MATERIALS AND METHODS}

\section{Animals and experimental design}

The study was approved by the Institutional Animal
Care and Use Committee, Hanyang University College of Medicine, in accordance with the Guide for the Care and Use of Laboratory Animals published by the National Institutes of Health and was performed in accordance with approved guidelines (no. 2018-0053A).

We created a renal IRI model using 8-week-old male Sprague-Dawley rats (SD rats; OrientBio, Seongnam, Korea). Rats were divided into three groups. Animals in the sham group ( $\mathrm{n}=5$ /group) underwent right nephrectomy; ischemia was not induced, and the abdominal cavity was opened for the usual duration of ischemia and then sutured and closed. Animals in the IRI group ( $\mathrm{n}=5$ /group) underwent right nephrectomy, and a bulldog clamp was used to occlude the left renal artery and vein. Animals in the CORM-3 group ( $\mathrm{n}=5 /$ group) were injected with CORM-3 $(10 \mathrm{mg} / \mathrm{kg})$ in the tail vein 1 hour preoperatively and were then subjected to the same procedure as those in the IRI group.

The detailed surgical procedures are presented in Supplementary material.

\section{TUNEL assay}

Renal tissue that had been stored and fixed in $4 \%$ formaldehyde was treated with ethanol and xylene, and paraffinembedded samples were cut into $4-\mu \mathrm{m}$-thick sections and prepared as slides. After using xylene to remove the paraffin, the tissue was rehydrated using $100 \%, 90 \%, 80 \%$, and $70 \%$ ethanol. The renal tissue was then soaked in Proteinase K solution $(20 \mu \mathrm{g} / \mathrm{mL})$ prepared in $10 \mathrm{mM}$ Tris/HCl $(\mathrm{pH}$ $7.4-8)$, incubated for 15 minutes at room temperature $\left(25^{\circ} \mathrm{C}\right.$ to $27^{\circ} \mathrm{C}$ ), and washed with phosphate-buffered saline (PBS). We used the In Situ Cell Death Detection Kit, Fluorescein (Roche, Basel, Switzerland) to prepare a mixture of the enzyme solution $(5 \mu \mathrm{L})$ and label solution $(45 \mu \mathrm{L})$. The prepared mixture $(50 \mu \mathrm{L})$ was dripped onto the renal tissue, which was then covered with a cover slip. The slides were left to react at $37^{\circ} \mathrm{C}$ for 1 hour and were washed with PBS after the completion of the reaction. Finally, the kidney tissue was soaked in a $1 \mu \mathrm{g} / \mathrm{mL}$ Hoechst 33342 (2'-4-ethoxyphenyl]-5[4methyl-1-piperazinyl]-2,5'-bi-1H-benzimidazole trihydrochloride trihydrate; Thermo Scientific, Waltham, MA, USA) solution prepared in PBS and left to react for 15 minutes at room temperature. The tissue was washed with PBS and mounted using VECTASHIELD Antifade Mounting Medium (Vector Laboratories, Burlingame, CA, USA). Areas of cell death were identified as green fluorescence in the cellular nuclei (stained in blue) under a light microscope (Leica Microsystems, Wetzar, Germany). 


\section{Picro-sirius red staining}

Xylene was used to remove paraffin from $4-\mu \mathrm{m}$-thick renal tissue slides, and the tissue was rehydrated in $100 \%, 90 \%$, $80 \%$, and $70 \%$ ethanol and washed with distilled water (DW). The tissue was stained with Picro-sirius red solution (Abcam, Cambridge, UK) for 1 hour at room temperature. Thereafter, it was washed twice in $0.5 \%$ acetic acid solution; hydrated in $70 \%, 80 \%, 90 \%$, and $100 \%$ ethanol; treated with xylene; and mounted using Consul-Mount Histology Formulation (Thermo Scientific).

\section{Immunohistochemical staining}

Xylene was used to remove paraffin from $4-\mu$ m-thick renal tissue slides, and the tissue was rehydrated in $100 \%, 90 \%$, $80 \%$, and $70 \%$ ethanol and washed with DW. The slides were treated with sodium citrate buffer ( $\mathrm{pH}$ 6.0), placed in a microwave, and heated for 15 minutes for antigen retrieval. After dripping 0.3\% Triton X-100/PBS onto the kidney tissue, the tissue was left to react for 10 minutes at room temperature and washed with PBS. For blocking, the kidney tissue was treated with 10\% normal donkey serum (Abcam, Cambridge, MA, USA), diluted in PBS, for 1 hour at room temperature. After blocking, the tissue was treated with the primary antibody (KIM-1; R\&D Systems, Minneapolis, MN, USA) diluted in PBS. After completion of the antibody reaction, the tissue was washed in PBS and diaminobenzidine (DAB) staining was performed. The renal tissue was reacted with the DAB mixture (DAB stock, $100 \mu \mathrm{L}$; Buffer stock, $84 \mu \mathrm{L}$; hydrogen peroxide, $80 \mu \mathrm{L}$; and DW) prepared by following the manual of the DAB Substrate Kit (Vector Laboratories). Thereafter, the tissue was washed in DW and briefly immersed in hematoxylin staining solution 20 times for nuclear staining. The tissue was hydrated using 70\%, 80\%, 90\%, and $100 \%$ ethanol and mounted using the Consul-Mount Histology Formulation (Thermo Scientific).

\section{CDNA microarray}

For each sample, $100 \mathrm{ng}$ of RNA was amplified and labeled with Cy3-dCTP. The labeled cRNAs were purified using the RNAeasy Mini Kit (Qiagen, Hilden, Germany), and the concentration of labeled cRNAs (pmol Cy3/. $\mathrm{g}$ cRNA) was measured using an ND-1000 Spectrophotometer. cRNAs (600 ng) were added to $5 \mu \mathrm{L}$ of $10 \times$ blocking agent and $1 \mu \mathrm{L}$ of $25 \times$ fragmentation buffer, and the mixture was heated for 30 minutes at $60^{\circ} \mathrm{C}$. Finally, cRNAs were diluted in $25 \mu \mathrm{L}$ of $2 \times \mathrm{GE}$ hybridization buffer. After $40 \mu \mathrm{L}$ of hybridization solution was transferred to gasket slides, the slides were assembled into Agilent SurePrint G3 Human GE 8X60K, V3 Microarrays (Agilent, Santa Clara, CA, USA). Raw data were obtained using Agilent Feature Extraction Sof tware (v11.0.1.1) and summarized in the Agilent feature extraction protocol to produce a raw data text file. The Flag A-tagged array probes were filtered. The selected gProcessed signal value was subjected to logarithmic transformation and normalized using the quantile method. The statistical significance of the expression data was determined by the fold change between the two groups and was verified by an independent t-test. Hierarchical cluster analysis was performed using complete linkage and Euclidean distance as measures of similarity. Gene Ontology (http://geneontology.org) and KEGG (http:// kegg.jp) were used to perform gene-enrichment and functional annotation analysis of the probe list. Data analyses and visualization of genes showing significant differences were performed using R3.0.2 (www.r-project.org).

\section{RESULTS}

\section{Changes in blood creatinine levels}

Blood creatinine levels were measured to assess in vivo renal function (Fig. 1). The sham group showed no significant changes in blood creatinine levels; however, the IRI group showed a gradual increase in the blood creatinine level in the 60-minute ischemia model and a large increase in the 75-minute ischemia model. The CORM-3 group showed lower blood creatinine levels after 75 minutes of ischemia than in the IRI group, which was not administered CORM-3 (1.2 vs. $2.4 \mathrm{mg} / \mathrm{dL}, \mathrm{p}=0.01)$. The 75-minute ischemia model was selected for further experiments because it showed the largest difference in the blood creatinine level.

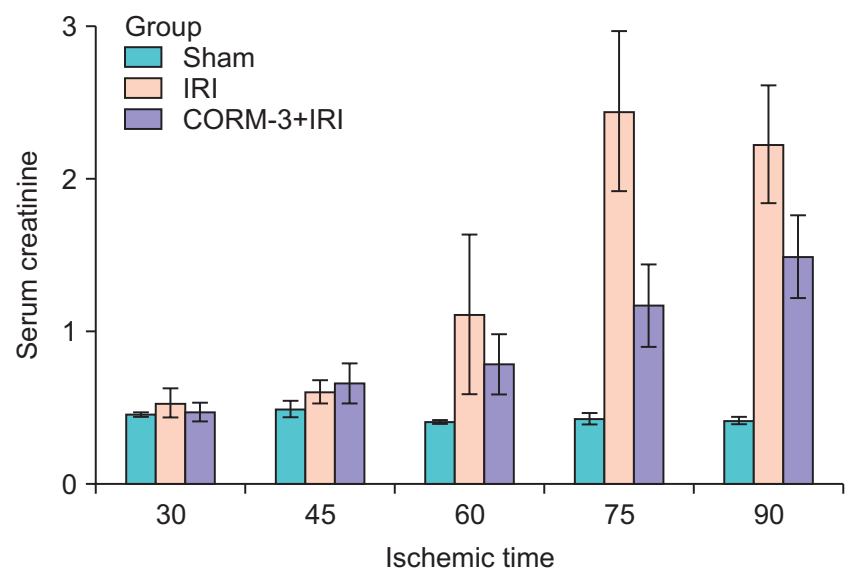

Fig. 1. Serum creatinine concentrations at 1 day after ischemia-reperfusion injury (IRI). In the 30-minute and 45-minute IRI experiments, there were no significant differences among the groups, but the effect of carbon monoxide-releasing molecule-3 (CORM-3) was seen in IRI injuries of more than 60 minutes. 


\section{Changes in the level of renal injury markers}

We measured blood levels of the renal injury markers KIM-1, cystatin-C, and beta 2-microglobulin. Blood concentrations of all three markers were higher in the IRI group than in the sham group. KIM-1 and cystatin-C levels were significantly lower in the CORM-3 group than in the IRI group (550.4 \pm 55.9 vs. $292 \pm 68.6 \mathrm{pg} / \mathrm{mL}$, p<0.001, and 3,933.7 \pm 106.1 vs. $3,324.9 \pm 309.8 \mathrm{ng} / \mathrm{mL}, \mathrm{p}=0.01$, respectively). Furthermore, the level of beta 2-microglobulin was lower in the CORM3 group than in the IRI group ( $p=0.116$, Fig. 2 ), although the difference was not statistically significant.

\section{Histologic tests (hematoxylin and eosin staining, TUNEL assay, and immunohistochemical assay)}

We used histologic tests in experimental animals to determine changes in tissue morphology caused by IRI and CORM-3. Hematoxylin and eosin staining revealed normal tubule appearance of the kidney tissue in the sham group (Fig. 3). However, the IRI group exhibited severe tubular dilatation (open lumen) and tubular epithelial thinning. Moreover, extensive accumulation of intraluminal cytoplasmic debris and necrosis of the tubular epithelium were observed at both high $(\times 400)$ and low $(\times 100)$ magnification. Conversely, the CORM-3 group showed only partial tubular thinning, and no necrosis was observed.

The TUNEL assay was performed to determine apoptotic activity in the kidney. The sham group exhibited few apoptotic cells; however, the level of apoptosis increased markedly in the IRI group, and CORM-3 administration reduced the number of apoptotic cells (Fig. 4). Immunohistochemistry was performed to determine the levels of KIM-1 in the renal tissue. As expected, the CORM-3 group showed lower KIM1 levels than in the IRI group ( $p=0.003$, Fig. 5). Furthermore, we used Picro-sirius red staining to examine the extent of fibrosis in the renal tissue. The CORM-3 group showed less fibrosis than in the IRI group; however, the difference was not statistically significant ( $p=0.115$, Fig. 6).

\section{4. cDNA microarray}

Of the 45,598 probes detected by cDNA microarray analysis, 3,820 showed significant differences. In the IRI group, 3,746 genes showed altered expression, while the expression of only 960 genes was altered in the CORM-3 group. As shown in the Venn diagram plots (Fig. 7), the genes with aberrant expression in the CORM-3 group were mostly concordant with those in the IRI group. We observed 1,379 and 1,481 genes that were upregulated and downregulated, respectively, in the IRI group. These results suggest that CORM-3
A
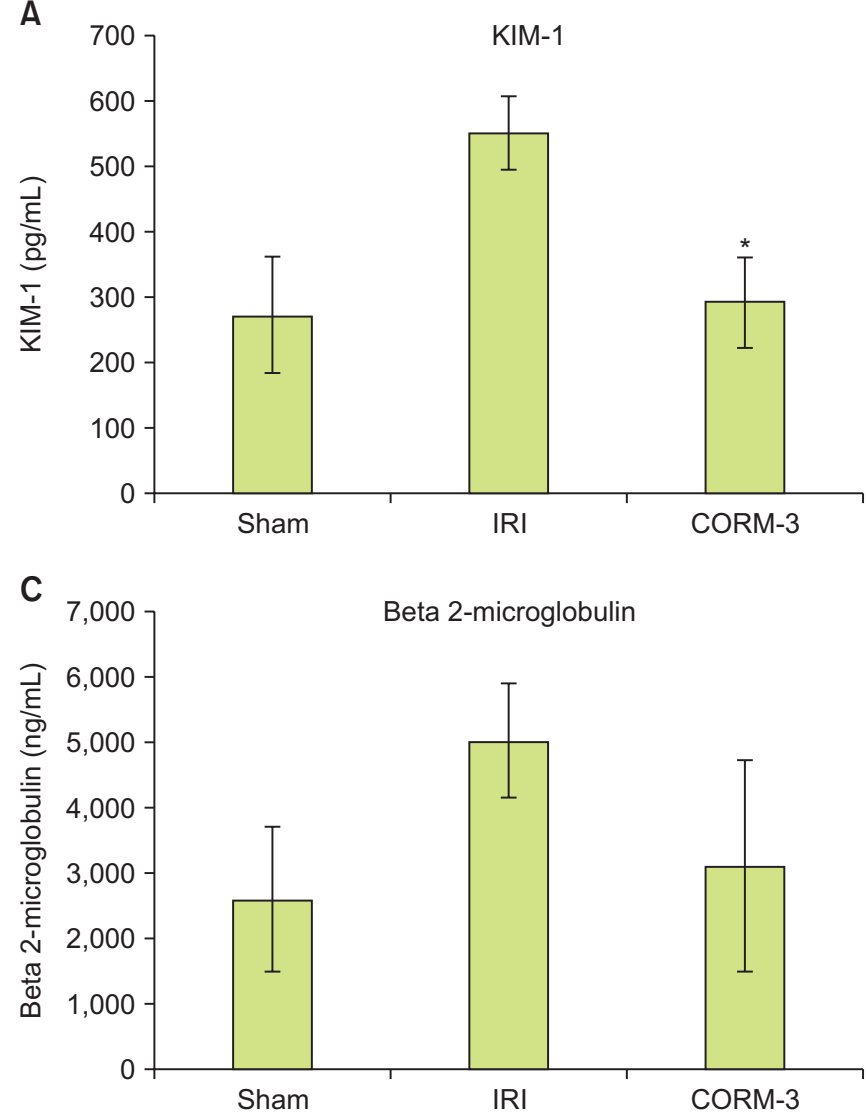

B

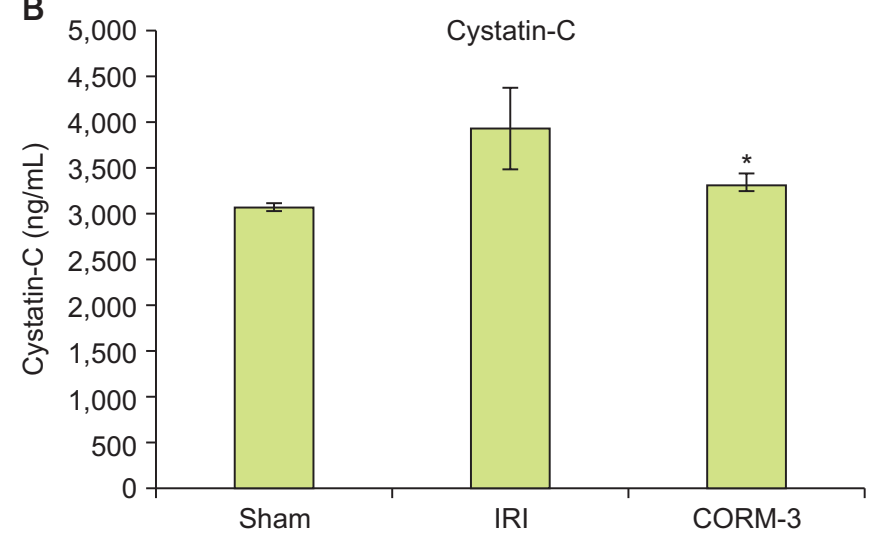

Fig. 2. (A-C) In vivo model of renal injury markers. ${ }^{*} p<0.05$ compared with ischemia-reperfusion injury (IRI) group. CORM-3, carbon monoxide-releasing molecule-3. 

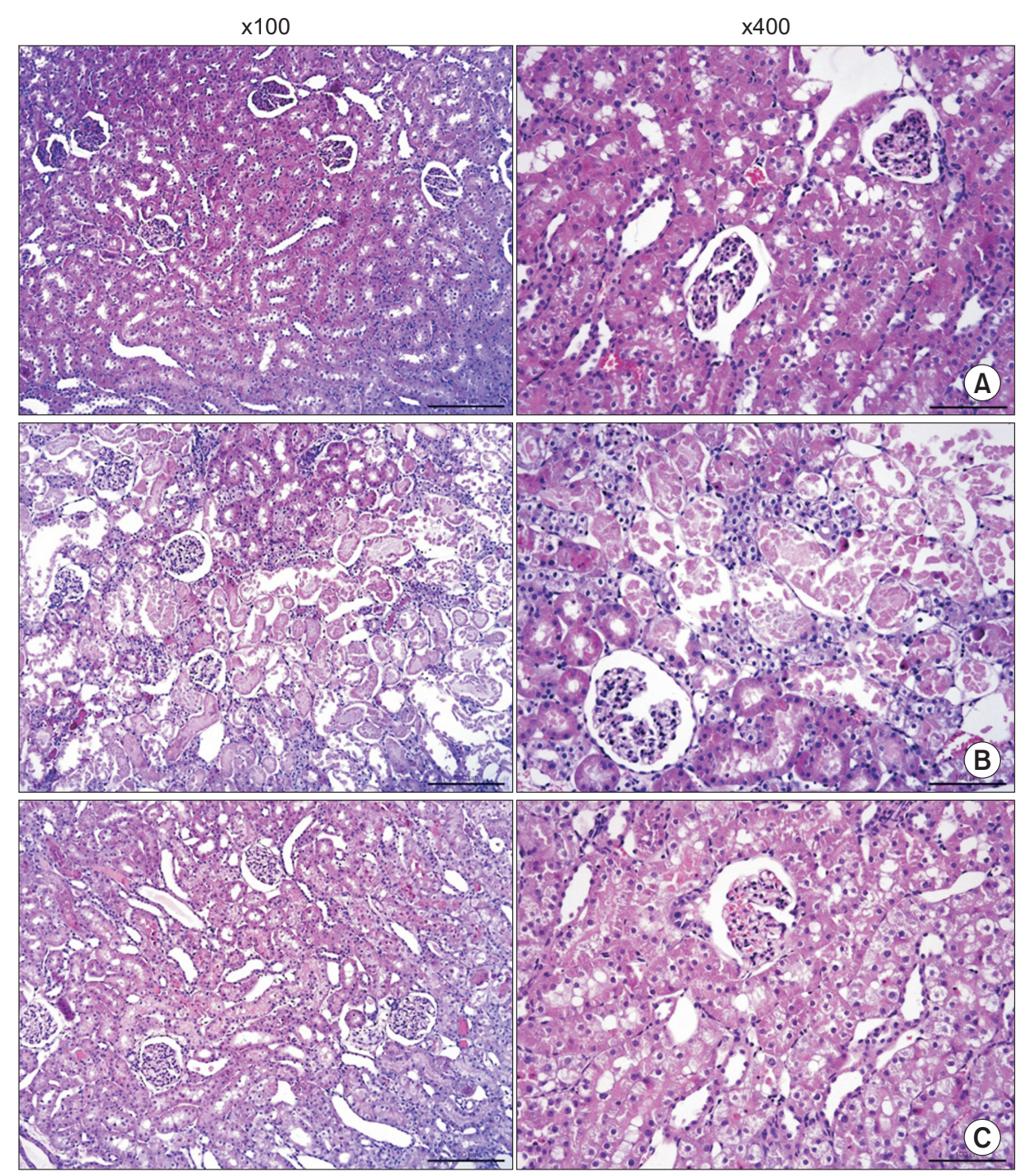

Fig. 3. Hematoxylin and eosin staining of kidney. (A) Sham, (B) ischemia-reperfusion injury group, and $(C)$ carbon monoxidereleasing molecule-3 group.

prevents changes in gene expression associated with IRI.

We also studied the pathways involving genes with aberrant expression in the IRI group and identified five pathways, including the metabolic and PI3K-Akt signaling pathways (Table 1). In the CORM-3 group, the expression of these genes was poor or not detected.

Differences in the expression of genes associated with the peroxisome proliferator-activated receptors (PPAR) signaling pathway, glycolysis, gluconeogenesis, amino acid biosynthesis, vascular smooth muscle contraction or relaxation, steroid hormone biosynthesis, and fatty acid metabolism were observed between the IRI and CORM-3 groups. A total of 15 genes were found to be involved in the PPAR signaling pathway (MCAD, LXR-alpha, SLC27A2, EHHADH, ACSL1, Perilipin-2, PPAR-alpha, CYP4A1, CYP4A2, CYP4A3, CYP4A8, HMGCS2, PEPCK1, RXR-gamma, and glycerol kinase), and the difference in the expression of these genes was statistically significant ( $p<0.001$, Fig. 8).
In order to analyze changes in the expression of genes involved in the PPAR signaling pathway in the sham, IRI, and CORM-3 groups, gene-specific primers were designed and reverse transcription polymerase chain reaction was performed. The expression of PPAR pathway genes was significantly lower in the IRI group than in the sham group. Moreover, the expression of PPAR pathway genes was higher in the CORM-3 group than in the IRI group (Fig. 9). These results suggest that pretreatment with CORM-3 may inhibit IRI by upregulating genes associated with the PPAR signaling pathway.

\section{DISCUSSION}

The aim of this study was to demonstrate by use of a rat model the ability of CORM-3 to prevent and treat renal IRI. We showed that CORM-3 pretreatment can reduce cell death and inflammatory responses after IRI and can increase cell 
DAPI

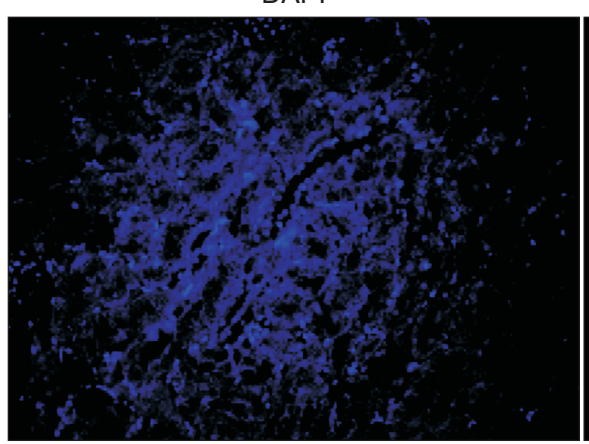

TUNEL
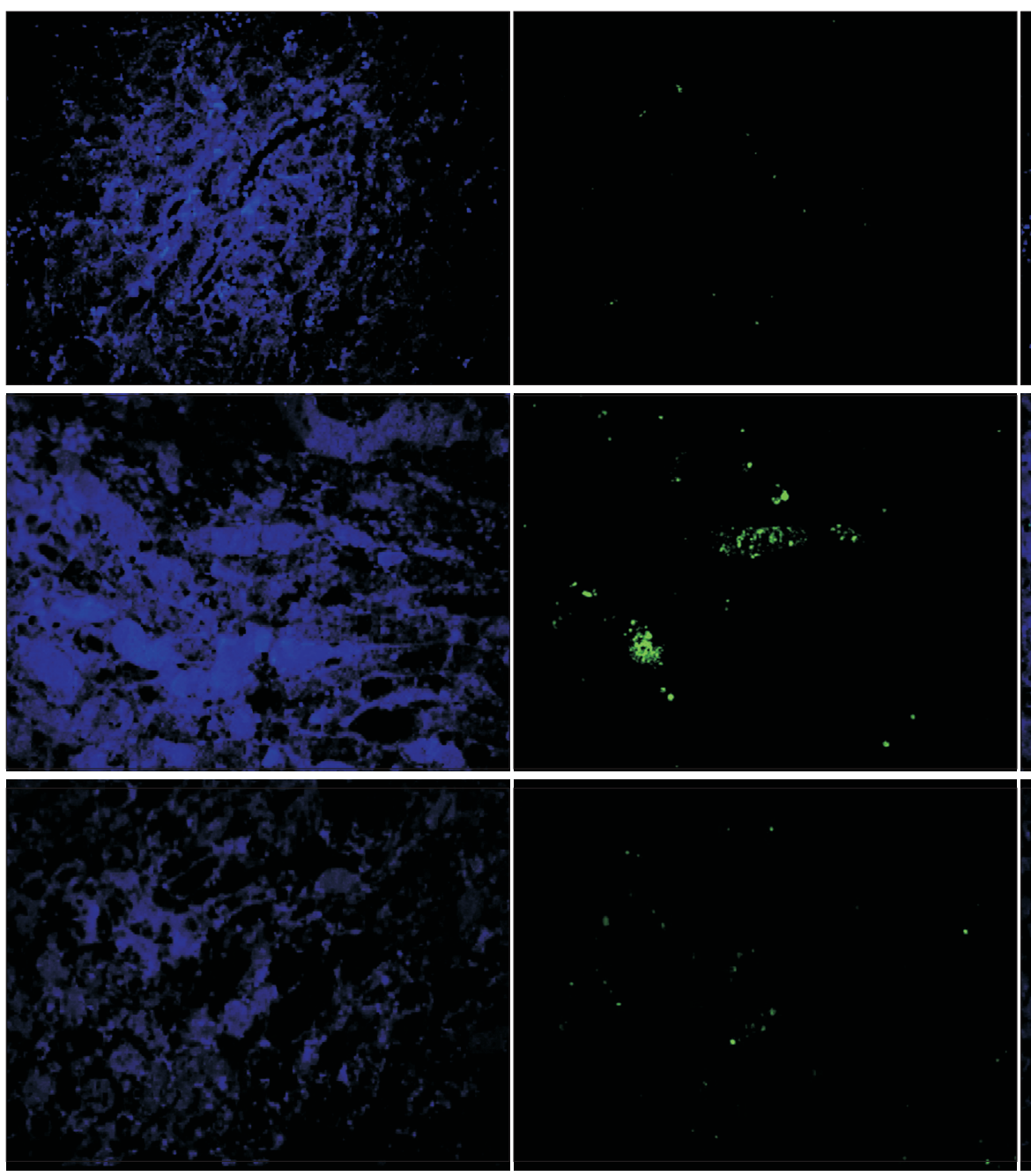

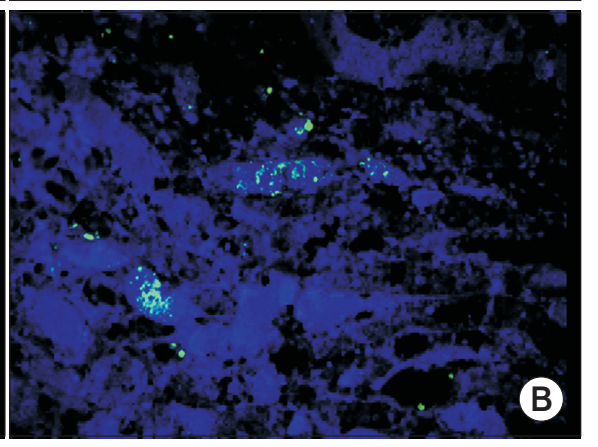

Merge
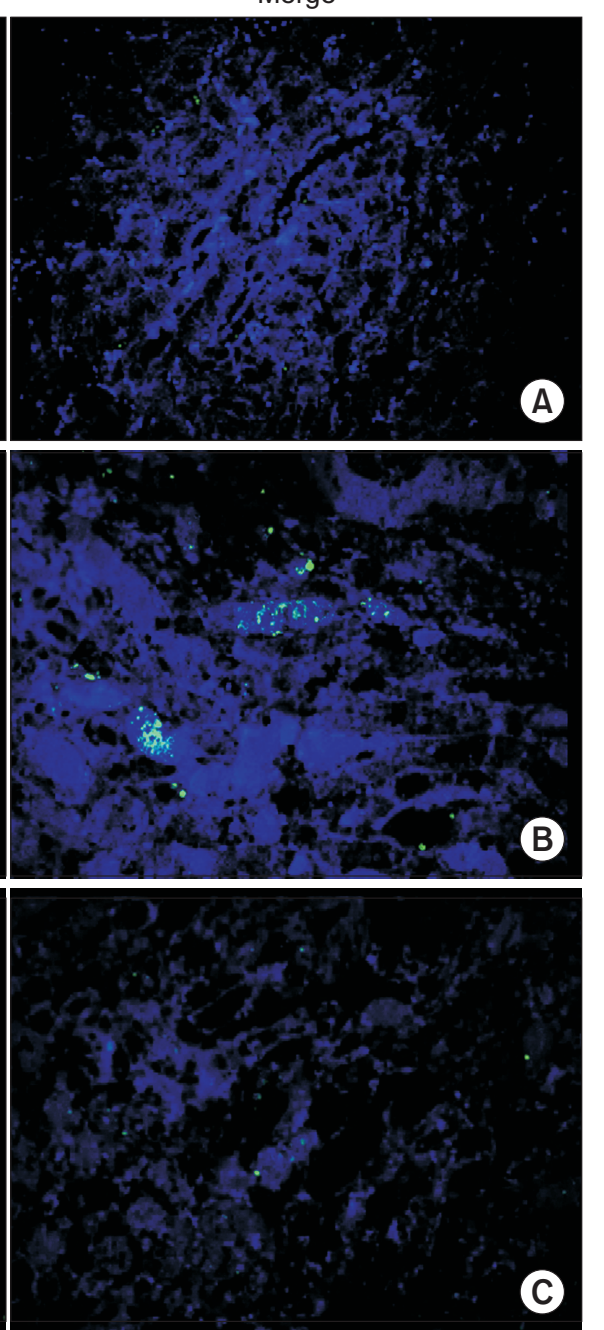

Fig. 4. TUNEL assay of kidney. (A) Sham, (B) ischemia-reperfusion injury group, and (C) carbon monoxide-releasing molecule-3 group.

growth. The results of the immunohistochemistry, pathologic examination, and cDNA microarray analyses showed that CORM-3 pretreatment can preserve renal function. CORM-3 reduces renal IRI through the upregulation of genes involved in the PPAR signaling pathway.

Several previous studies investigated mechanisms to prevent or minimize renal IRI. One study developed a model to minimize IRI by using ischemic preconditioning with bradykinins, antioxidants, and inflammatory molecules. In that study, Yoon et al. [11] conducted an in vivo study of remote ischemic preconditioning to determine its underlying mechanism. When applied in a clinical setting to partial nephrectomy for patients with a renal mass, because of the difficulties in actually performing two rounds of anesthesia and surgery, chemical treatment methods for renal IRI have been suggested. Studies conducted in the 2010s involved pretreatment with sildenafil, udenafil, or L-arginine and $\alpha$-tocopherol to treat IRI [12-14]. These methods were reported to alleviate IRI by increasing renal blood flow and reducing neutrophil activity, reactive oxygen species levels, and platelet aggregation. Subsequent studies used hydrogen sulfide to treat IRI in renal transplantation patients. The vasodilatory effect of hydrogen sulfide on microvascular flow was enhanced by concomitant administration of $\mathrm{CO}$. However, the role of hydrogen sulfide in inflammatory modulation is still debated; hydrogen sulfide has also been shown to aggravate sepsis owing to neutrophil migration $[15,16]$.

Interestingly, $\mathrm{CO}$ has been reported to play a protective role in a rodent kidney warm IRI model [17,18]. CO was initially used for inhalation therapy. CO gas is relatively nonreactive and is simple to administer, with well-described pharmacodynamics. Low-dose CO inhalation therapy has been used in clinical practice in pulmonary and vascular diseases such as pulmonary fibrosis, ventilator-induced lung injury, and diabetes. CORM, unlike CO gas inhalation, has the potential to reduce excessive exposure through tissue- 


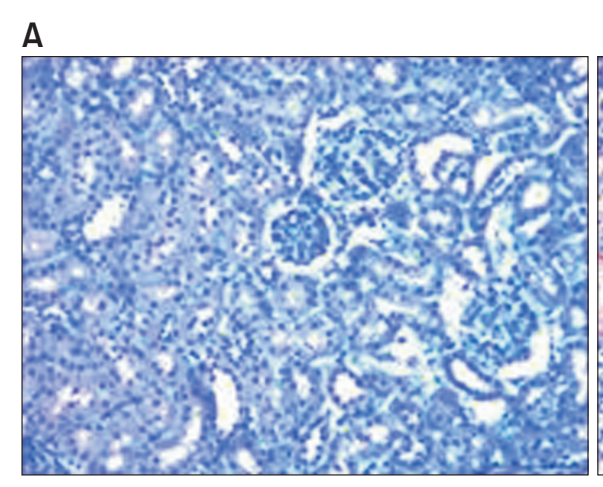

B

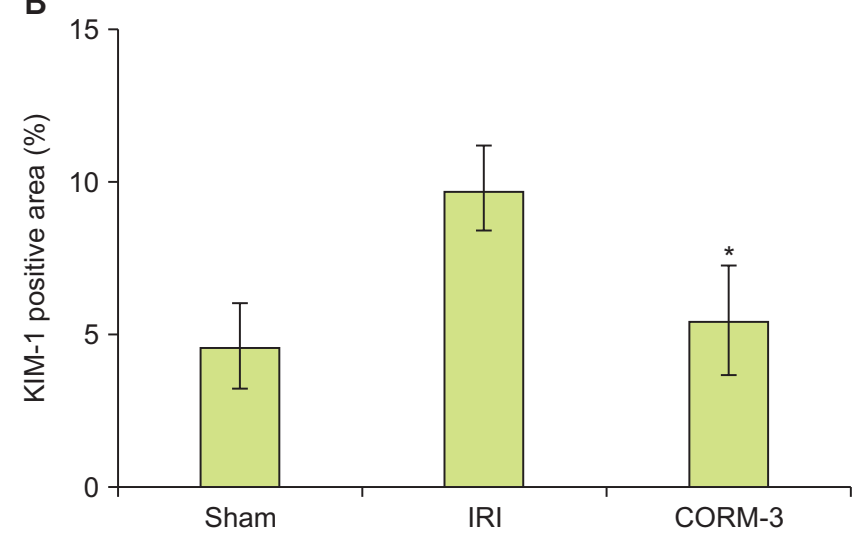

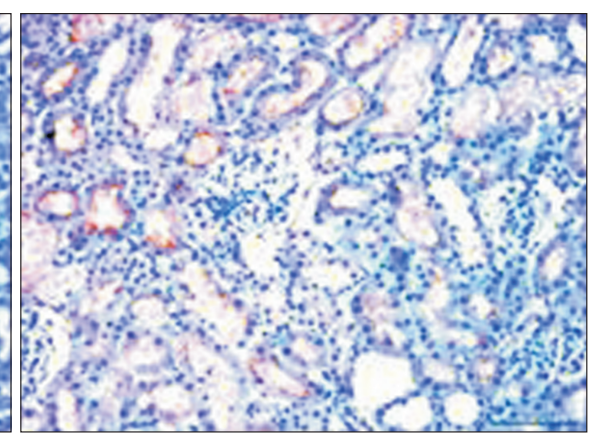

IRI

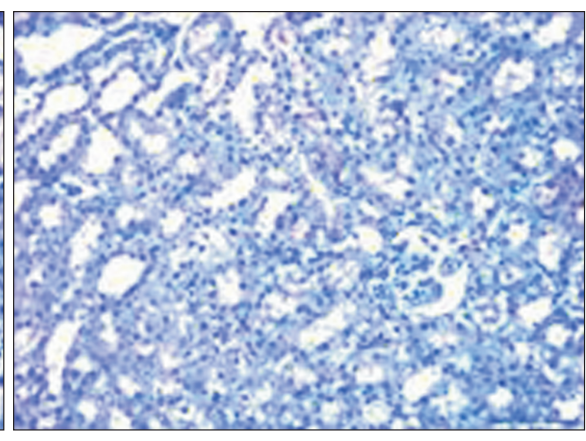

CORM-3
Fig. 5. (A, B) Immunohistochemistry of kidney injury molecule-1 $(\times 400) .{ }^{*} p=0.003$ compared with ischemia-reperfusion injury (IRI) group. CORM-3, carbon monoxide-releasing molecule-3.

A

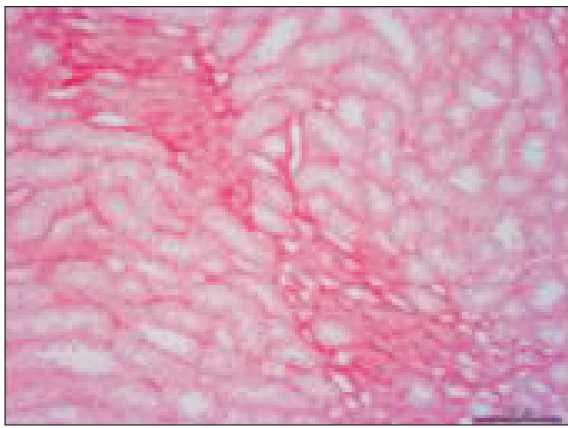

Sham

B

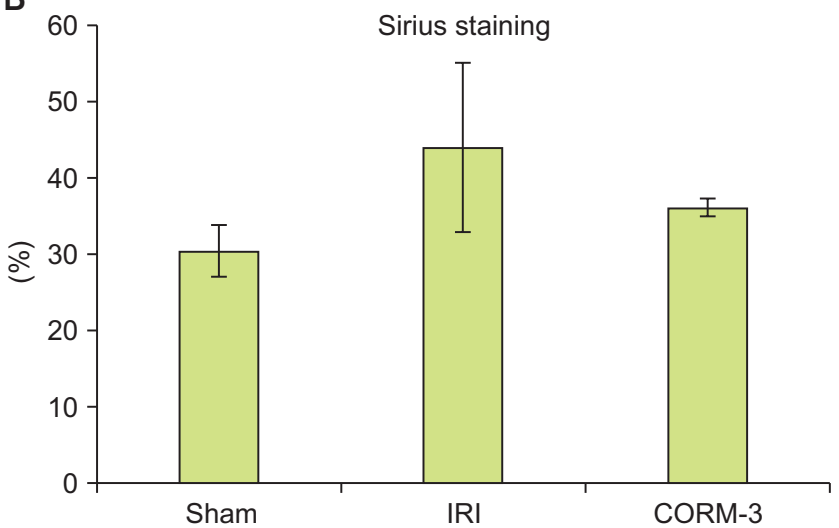

specific delivery and through a range of $\mathrm{CO}$ release kinetics. Additionally, CORM can be used to deliver small amounts of $\mathrm{CO}$ to biological systems in a controlled manner and could

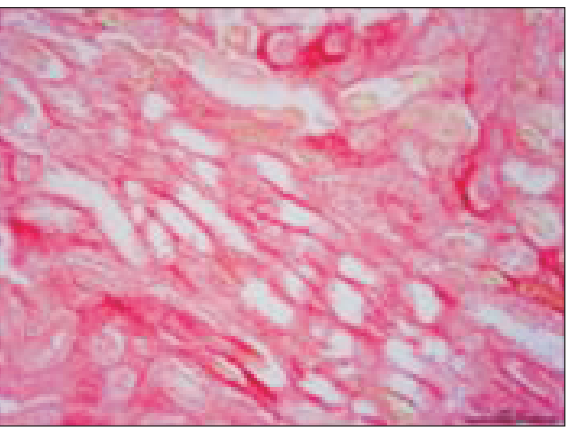

IRI

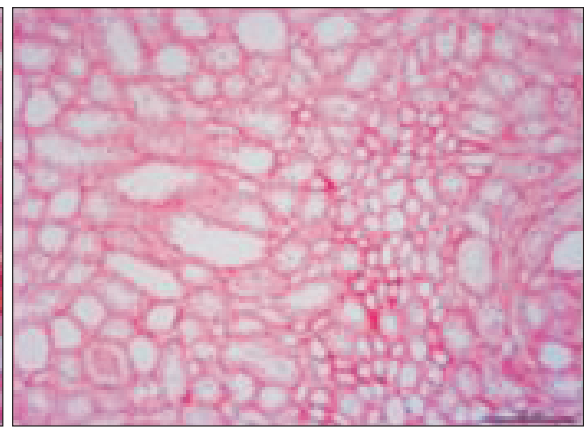

CORM-3

Fig. 6. (A, B) Sirius red staining of kidney $(\times 400)$. IRI, ischemia-reperfusion injury; CORM-3, carbon monoxide-releasing molecule-3.

deliver $\mathrm{CO}$ to tissues with less $\mathrm{COHb}$ compared with inhaled CO [10,19]. Recent research has demonstrated that metal carbonyl compounds can act as specific CO donors [20]. CORM- 


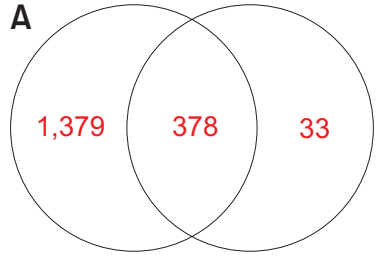

IRI CORM-3

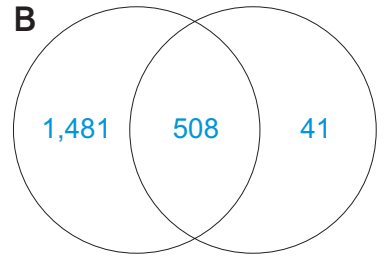

IRI CORM-3
Fig. 7. Venn diagrams of altered gene expression. (A) Upregulated by two-fold or more, (B) downregulated by two-fold or more. IRI, ischemia-reperfusion injury; CORM-3, carbon monoxide-releasing molecule-3.
Table 1. Genes of the KEGG pathway with altered expression in the ischemia-reperfusion injury (IRI) and carbon monoxide-releasing molecule-3 (CORM-3) groups

\begin{tabular}{lcc}
\hline \multirow{2}{*}{ KEGG pathway } & \multicolumn{2}{c}{ Altered genes } \\
\cline { 2 - 3 } & IRI & CORM-3 \\
\hline Metabolic pathways & 346 & 93 \\
PI3K-Akt signaling pathway & 70 & 21 \\
Oxidative phosphorylation & 53 & $\mathrm{~N} / \mathrm{A}$ \\
Carbon metabolism & 50 & 17 \\
Cell cycle & 39 & 25 \\
\hline
\end{tabular}

N/A, not applicable.

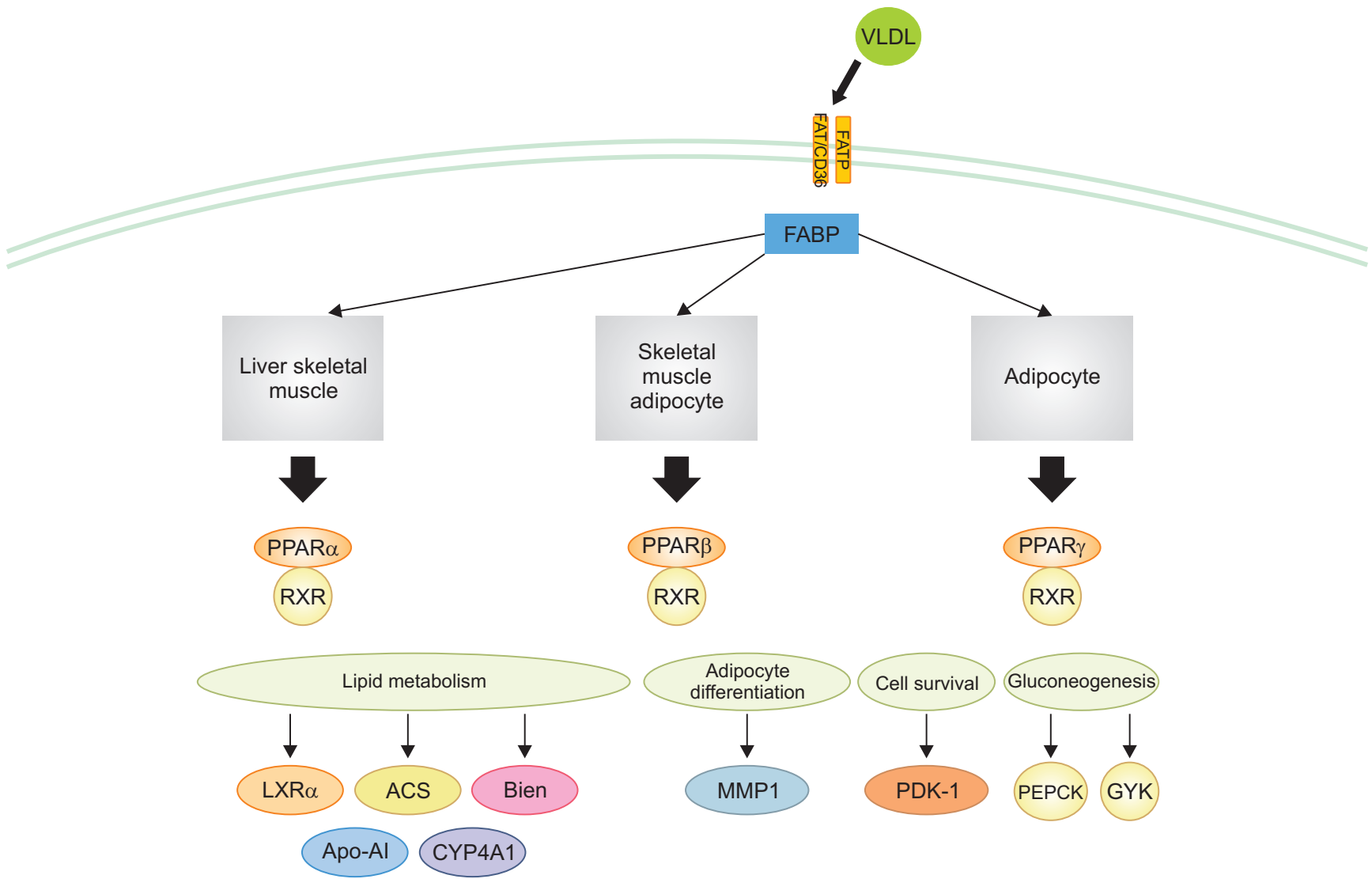

Fig. 8. Genes in the peroxisome proliferator-activated receptors (PPAR) signaling pathway with altered expression in the carbon monoxide-releasing molecule-3 group compared with the ischemia-reperfusion injury group. VLDL, very low density lipoprotein; FATP, fatty acid transport proteins; FAT/CD36, fatty acid translocase/CD36; FABP, fatty acid binding protein; RXR, retinoid X receptor; ACS, acyl-CoA synthetase; Bien, bifunctional enzyme; MMP1, matrix metalloproteinase-1; PDK-1, pyruvate dehydrogenase kinase 1; PEPCK, phosphoenolpyruvate carboxykinase; GYK, glycerol kinase; Apo-Al, apolipoprotein A-l; CYP4A1, cytochrome P450, family 4, subfamily a.

1 and CORM-2 are hydrophobic. In this study, CORM-3 $[\mathrm{Ru}(\mathrm{CO}) 3 \mathrm{Cl}($ glycinato)] was used, which has excellent water solubility at $\mathrm{pH} 3$ and rapidly releases $\mathrm{CO}$ in physiologic fluids. CORM-3 was administered intravenously 1 hour before IRI. No studies have yet been conducted to determine the most effective time of CORM-3 administration before ischemia. Further research will be required to investigate the optimal time of administration of CORM-3.

There have been several efforts in the urology field to elucidate the genes related to the IRI pathway [21]. Toll-like receptors, sphingosine-1-phosphate receptors, and hypoxiainducible factors have been reported to worsen tubular epithelial cell function, eventually causing acute renal damage, delayed graft function, and ultimately acute-on-chronic rejection [22]. In this study, metabolic pathways, the PI3KAkt signaling pathway, oxidative phosphorylation, carbon metabolism, and the cell cycle were found to be involved in renal IRI. Moreover, only a few genes in the CORM-3 group 


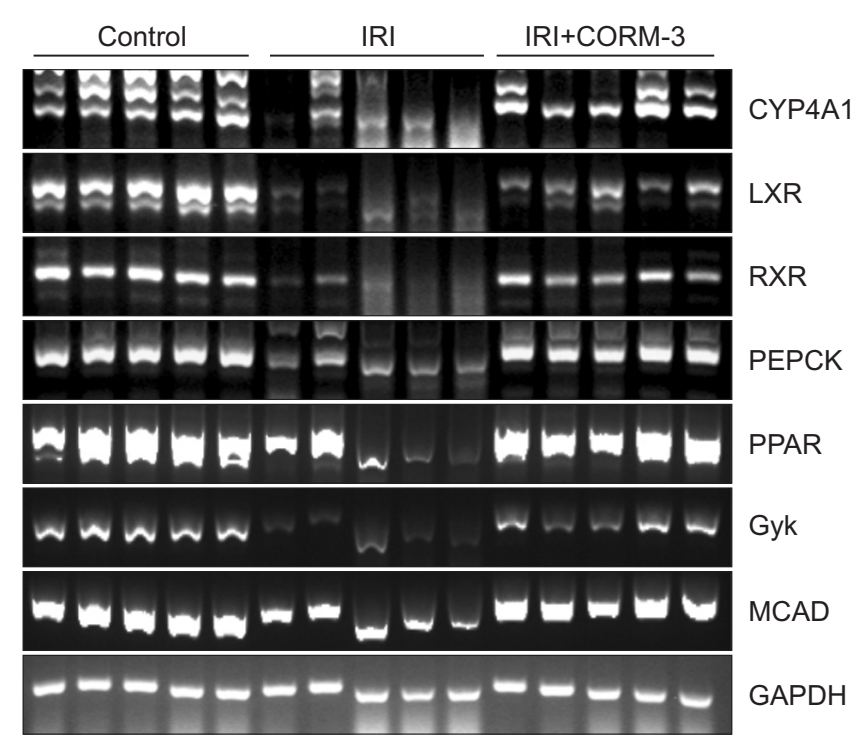

Fig. 9. Identification of changes in gene expression related to the peroxisome proliferator-activated receptors (PPAR) signaling pathway. IRI, ischemia-reperfusion injury; CORM-3, carbon monoxide-releasing molecule-3; CYP4A1, cytochrome P450, family 4, subfamily a; LXR, liver $X$ receptor; $R X R$, retinoid $X$ receptor; $P E P C K$, phosphoenolpyruvate carboxykinase; Gyk, glycerol kinase; MCAD, medium chain acyl dehydrogenase; GAPDH, glyceraldehyde 3-phosphate dehydrogenase.

showed altered expression. We hypothesize that CORM-3 prevents changes in gene expression by protecting against renal IRI. Indeed, the CORM-3 group showed significantly less gene expression than the IRI group.

The PPAR signaling pathway is involved in lipid metabolism, gluconeogenesis, and cell survival. PPAR is of interest owing to its protective effect on IRI. Although the effects of PPAR in cardiac, cerebral, hepatic, and renal IRI have already been studied, the precise role of PPAR and its potential as a therapeutic agent remain relatively underresearched [23-25]. However, various experimental studies have shown PPAR to be involved in renal IRI. PPAR agonists have been shown to reduce IRI [26-28]. Although there is no direct evidence of the role of PPAR- $\delta$ in renal IRI, a study has demonstrated the agonist activity of PPAR- $\gamma$ and PPAR- $\delta$ [29]. In this present study, we described the potential role of PPAR in the mechanism of CORM-3. However, further research is warranted to validate our findings.

A limitation of this study is that we could not perform experiments using larger animals that closely resemble ischemic injury in humans. In order to use CORM-3 as a treatment for IRI, safety assessment in humans will be necessary. Additionally, a negative control group using an inactive form of CORM-3 was not investigated. Nevertheless, our in vivo experiments using rats demonstrated that CORM-3 has potential for use as a pretreatment agent in laparoscopic or robotic partial nephrectomy to cause warm IRI. To our knowledge, this is the first study providing in vivo evidence supporting the use of CORM-3 in humans.

\section{CONCLUSIONS}

In conclusion, CORM-3 inhibits the progression of acute renal injury due to IRI by reducing inflammation and renal cell death. Moreover, CORM-3 reduces the inflammatory response of renal cells and suppresses apoptosis. The PPAR pathway suppresses responses associated with cell death and plays an important role in the mechanism of CORM-3. Through further human studies, we expect that pretreatment with CORM-3 in partial nephrectomy and kidney transplantation will minimize renal IRI.

\section{CONFLICTS OF INTEREST}

The authors have nothing to disclose.

\section{ACKNOWLEDGMENTS}

This research was supported by the National Research Foundation of Korea (NRF) grant funded by the Korea government (MSIT) (NRF-2017R1C1B5018097). We thank A Ra Jung for contributing animal surgery and technical assistance. This manuscript was selected as the best paper at the 71st Korean Urological Association meeting in 2019.

\section{AUTHORS' CONTRIBUTIONS}

Research conception and design: Young Eun Yoon and Hong Sang Moon. Data acquisition: Dae Keun Kim. Statistical analysis: Young Eun Yoon and Jiyoung Lee. Data analysis and interpretation: Su-Jin Shin, Jiyoung Lee, and Dae Keun Kim. Drafting of the manuscript: Dae Keun Kim and Young Eun Yoon. Critical revision of the manuscript: Hong Sang Moon and Hong Yong Choi. Administrative, technical, or material support: Sung Yul Park and Yong Tae Kim. Supervision: Hong Yong Choi. Approval of the final manuscript: Hong Sang Moon.

\section{SUPPLEMENTARY MATERIAL}

Scan this QR code to see the supplementary material, or visit https://www.icurology.org/src/sm/icurology-61-441-s001.pdf. 


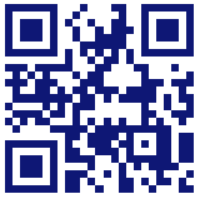

\section{REFERENCES}

1. Dowlatshahi K, Ajami M, Pazoki-Toroudi H, Hajimiresmaiel SJ. ATP-dependent potassium channels are implicated in simvastatin pretreatment-induced inhibition of apoptotic cell death after renal ischemia/reperfusion injury. Med J Islam Repub Iran 2015;29:191.

2. Hruby S, Lusuardi L, Jeschke S, Janetschek G. Cooling mechanisms in laparoscopic partial nephrectomy: are really necessary? Arch Esp Urol 2013;66:139-45.

3. Zargar H, Autorino R, Akca O, Brandao LF, Laydner H, Kaouk J. Minimally invasive partial nephrectomy in the age of the 'trifecta'. BJU Int 2015;116:505-6.

4. Thompson RH, Lane BR, Lohse CM, Leibovich BC, Fergany A, Frank I, et al. Every minute counts when the renal hilum is clamped during partial nephrectomy. Eur Urol 2010;58:340-5.

5. Hung AJ, Cai J, Simmons MN, Gill IS. “Trifecta” in partial nephrectomy. J Urol 2013;189:36-42.

6. Tingle SJ, Figueiredo RS, Moir JA, Goodfellow M, Talbot D, Wilson $\mathrm{CH}$. Machine perfusion preservation versus static cold storage for deceased donor kidney transplantation. Cochrane Database Syst Rev 2019;(3):CD011671.

7. Faleo G, Neto JS, Kohmoto J, Tomiyama K, Shimizu H, Takahashi $\mathrm{T}$, et al. Carbon monoxide ameliorates renal cold ischemiareperfusion injury with an upregulation of vascular endothelial growth factor by activation of hypoxia-inducible factor. Transplantation 2008;85:1833-40.

8. Hanto DW, Maki T, Yoon MH, Csizmadia E, Chin BY, Gallo $\mathrm{D}$, et al. Intraoperative administration of inhaled carbon monoxide reduces delayed graft function in kidney allografts in Swine. Am J Transplant 2010;10:2421-30.

9. Motterlini R, Mann BE, Foresti R. Therapeutic applications of carbon monoxide-releasing molecules. Expert Opin Investig Drugs 2005;14:1305-18.

10. Motterlini R, Otterbein LE. The therapeutic potential of carbon monoxide. Nat Rev Drug Discov 2010;9:728-43.

11. Yoon YE, Choi KH, Kim SY, Cho YI, Lee KS, Kim KH, et al. Renoprotective mechanism of remote ischemic preconditioning based on transcriptomic analysis in a porcine renal ischemia reperfusion injury model. PLoS One 2015;10:e0141099.

12. Lledo-Garcia E, Subira-Rios D, Ogaya-Pinies G, Tejedor-Jorge A, Cañizo-Lopez JF, Hernandez-Fernandez C. Intravenous sildenafil as a preconditioning drug against hemodynamic consequences of warm ischemia-reperfusion on the kidney. J
Urol 2011;186:331-3.

13. Özlülerden Y, Toktaş C, Aybek H, Küçükatay V, Şen Türk N, Zumrutbas AE. The renoprotective effects of mannitol and udenafil in renal ischemia-reperfusion injury model. Investig Clin Urol 2017;58:289-95.

14. Shokeir AA, Barakat N, Hussein AA, Awadalla A, Abdel-Aziz $A$, Abo-Elenin $\mathrm{H}$. Role of combination of L-arginine and $\alpha$-tocopherol in renal transplantation ischaemia/reperfusion injury: a randomized controlled experimental study in a rat model. BJU Int 2011;108:612-8.

15. Zhu JX, Kalbfleisch M, Yang YX, Bihari R, Lobb I, Davison M, et al. Detrimental effects of prolonged warm renal ischaemiareperfusion injury are abrogated by supplemental hydrogen sulphide: an analysis using real-time intravital microscopy and polymerase chain reaction. BJU Int 2012;110(11 Pt C):E121827.

16. Lobb I, Davison M, Carter D, Liu W, Haig A, Gunaratnam $L$, et al. Hydrogen sulfide treatment mitigates renal allograft ischemia-reperfusion injury during cold storage and improves early transplant kidney function and survival following allogeneic renal transplantation. J Urol 2015;194:1806-15.

17. Vera T, Henegar JR, Drummond HA, Rimoldi JM, Stec DE. Protective effect of carbon monoxide-releasing compounds in ischemia-induced acute renal failure. J Am Soc Nephrol 2005; 16:950-8.

18. Caumartin Y, Stephen J, Deng JP, Lian D, Lan Z, Liu W, et al. Carbon monoxide-releasing molecules protect against ischemia-reperfusion injury during kidney transplantation. Kidney Int 2011;79:1080-9.

19. Ryter SW, Choi AM. Carbon monoxide: present and future indications for a medical gas. Korean J Intern Med 2013;28:12340.

20. Motterlini R, Mann BE, Johnson TR, Clark JE, Foresti R, Green CJ. Bioactivity and pharmacological actions of carbon monoxide-releasing molecules. Curr Pharm Des 2003;9:252539.

21. Lee T, Lim US, Kang DH, Jung HD, Kim H, Choi BH, et al. Near-normalized gene expression profiles in bladder with detrusor overactivity in rats with bladder outlet obstruction after deobstruction. Int Neurourol J 2017;21:247-58.

22. Smith SF, Hosgood SA, Nicholson ML. Ischemia-reperfusion injury in renal transplantation: 3 key signaling pathways in tubular epithelial cells. Kidney Int 2019;95:50-6.

23. Linares I, Farrokhi K, Echeverri J, Kaths JM, Kollmann D, Hamar M, et al. PPAR-gamma activation is associated with reduced liver ischemia-reperfusion injury and altered tissueresident macrophages polarization in a mouse model. PLoS One 2018;13:e0195212.

24. Zhong CB, Chen X, Zhou XY, Wang XB. The role of peroxi- 
some proliferator-activated receptor $\gamma$ in mediating cardioprotection against ischemia/reperfusion injury. J Cardiovasc Pharmacol Ther 2018;23:46-56.

25. Collino M, Patel NS, Thiemermann C. PPARs as new therapeutic targets for the treatment of cerebral ischemia/reperfusion injury. Ther Adv Cardiovasc Dis 2008;2:179-97.

26. Sambandam N, Morabito D, Wagg C, Finck BN, Kelly DP, Lopaschuk GD. Chronic activation of PPARalpha is detrimental to cardiac recovery after ischemia. Am J Physiol Heart Circ Physiol 2006;290:H87-95.

27. Cuzzocrea S, Pisano B, Dugo L, Ianaro A, Patel NS, Di Paola $\mathrm{R}$, et al. Rosiglitazone and 15-deoxy-Delta12,14-prostaglandin
$\mathrm{J} 2$, ligands of the peroxisome proliferator-activated receptorgamma (PPAR-gamma), reduce ischaemia/reperfusion injury of the gut. Br J Pharmacol 2003;140:366-76.

28. Muià C, Mazzon E, Crisafulli C, Di Paola R, Genovese T, Caputi AP, et al. ROLE of endogenous peroxisome proliferatoractivated receptor-alpha (PPAR-alpha) ligands in the development of gut ischemia and reperfusion in mice. Shock 2006;25:17-22.

29. Hellemans K, Michalik L, Dittie A, Knorr A, Rombouts K, De Jong J, et al. Peroxisome proliferator-activated receptor-beta signaling contributes to enhanced proliferation of hepatic stellate cells. Gastroenterology 2003;124:184-201. 